\title{
Mucopolysaccharidosis Type IIIB
}

National Cancer Institute

\section{Source}

National Cancer Institute. Mucopolysaccharidosis Type IIIB. NCI Thesaurus. Code C84898.

A rare autosomal recessive lysosomal storage disease caused by deficiency of the enzyme $\mathrm{N}$-acetyl-alpha-D-glucosaminidase. It is characterized by behavioral changes, sleep disturbances, and mental developmental delays. 